\title{
Skin paddle vascularity of free fibula flap - A study of 386 cases and a classification based on contribution from axial vessels of the leg
}

\author{
Prabha S. Yadav, Quazi G. Ahmad, Vinay K. Shankhdhar, G.I. Nambi \\ Plastic and Reconstructive Services, Department of Surgical Oncology, TATA Memorial Hospital, Parel, Mumbai, India
}

Address for correspondence: Dr. G.I. Nambi, Plastic and Reconstructive Microvascular Services, Comprehensive Cancer Center, Kovai Medical Center and Hospital, Avinashi Road, Coimbatore, Tamilnadu - 641 014, India. E-mail: nambi75@rediffmail.com

\section{ABSTRACT}

Objective: The skin paddle of the free fibula flap receives its vascular supply from septocutaneous perforators, musculocutaneous perforators or from both, and these perforators might originate from the peroneal or posterior tibial vessels or from both. The objective of this study was to classify the skin paddles based on the dominance of vascular contribution by these axial vessels through their different perforator systems. Materials and Methods: A retrospective analysis of 5-year data of 386 free fibula flaps used in oro-mandibular reconstruction was done and the skin paddle vascularity was studied. While majority of the skin paddles received their blood supply from the peroneal septocutaneous perforators, a few had their dominant supply from the soleus musculocutaneous perforators in addition to peroneal septocutaneous perforators. In few cases, the soleus musculocutaneous perforators were the sole source of blood supply to the skin paddle. The limitation in this study was the inability to augment the clinical observation with cadaveric study. Results: The skin paddle of the free fibula flap was classified into four different types ( $a-d$ ) based on the dominance of vascular contribution by axial vessels of the leg. Conclusion: The skin paddle of the free fibula flap has reliable blood supply, but a thorough knowledge of the variations in vascular pattern of the skin paddle is required especially to salvage the larger paddles used in the reconstruction complex oro-mandibular defects.

\section{KEY WORDS}

Free fibula flap; musculocutaneous perforators; septo cutaneous perforators; skin paddle; vascular supply

\section{INTRODUCTION}

\begin{tabular}{|l|l|}
\hline \multicolumn{2}{|c|}{ Access this article online } \\
\hline Quick Response Code: & Website: \\
\hline & www.ijps.org \\
\cline { 2 - 2 } & DOI: \\
\hline
\end{tabular}

he free fibula flap is the most commonly used osteocutaneous flap for composite reconstructions owing to its versatile characters, ${ }^{[1-7]}$ one of them being the utilisation of the lateral leg skin from which a large skin paddle can be devised. The blood supply to the skin paddle is derived from septocutaneous perforators, musculocutaneous perforators or from 
both, ${ }^{[8-11]}$ and they may have their origin in the peroneal, posterior tibial or even the popliteal vessels. While the septocutaneous perforators are more in the lower leg, the musculocutaneous perforators are more in the upper leg, and therefore it is prudent to preserve them especially while raising larger skin paddles. ${ }^{[10-13]}$ In this study, we present a classification of the free fibula flap skin paddles based on the dominant source of their vascular supply.

\section{MATERIALS AND METHODS}

Three hundred and eighty-six free fibula flaps were used in the reconstruction of complex oro-mandibular defects over a period of 5 years (Jan 2005 to Dec 2009). In all the cases, pre-operative hand doppler assessment of the skin paddle perforators was done and the number of signals was ranging from 1 to 7 . All the donor sites were covered partly with split skin grafts from the opposite thigh. We considered large skin paddles as those whose length extended between the proximal and distal osteotomy sites and width extending to or beyond $2.5 \mathrm{~cm}$ on both sides of the postero-lateral septum of the leg. As we always follow simultaneous two-team approach, we prefer to harvest large skin paddles so as to compensate for any additional skin requirement arising after revision of the primary tumour site following frozen section biopsy of the surgical specimen. Musculocutaneous perforators were dissected till their origin from the posterior tibial vessels only when there were no septocutaneous perforators or when they provided significant contribution to the skin

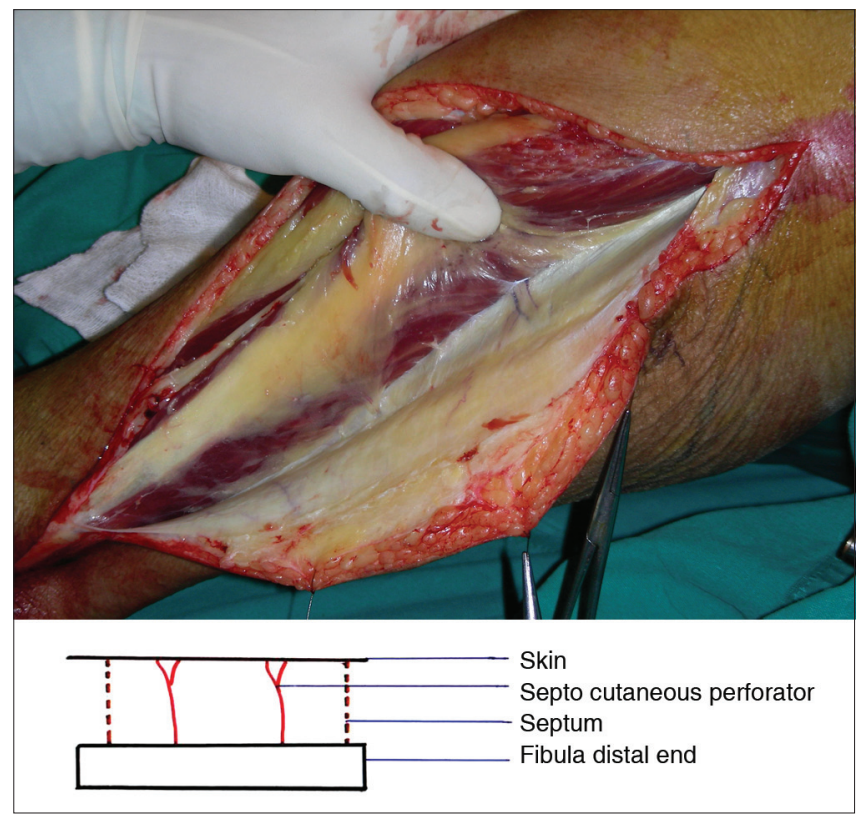

Figure 1: (a) Peroneal septocutaneous perforator supplying the skin paddle; (b) line diagram of Figure 1a paddle vascularity which was confirmed intraoperatively as described below. The data regarding the type of perforators contributing to skin paddle vascularity and the source of origin of these perforators alone were studied and classified as follows.

\section{Type A skin paddles}

Skin paddles receiving their vascular supply via the peroneal system alone were classified as type A skin paddles. They do not receive any contribution from other major axial vessels of the leg. In our series,

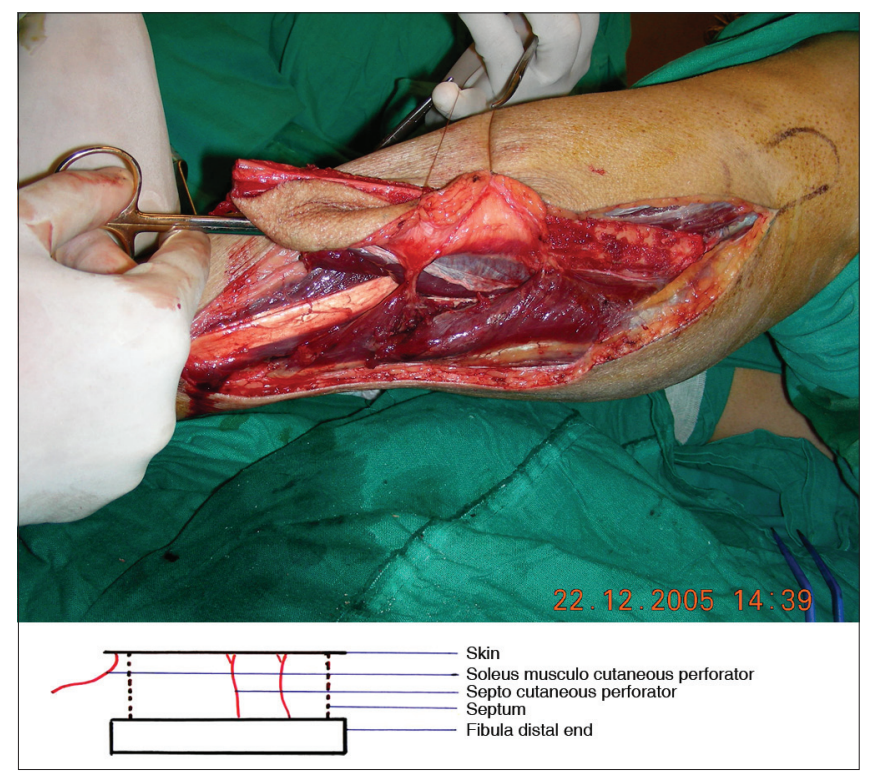

Figure 2: (a) Skin paddle receiving dominant contributions from soleus musculocutaneous perforator; (b) line diagram of Figure $2 a$

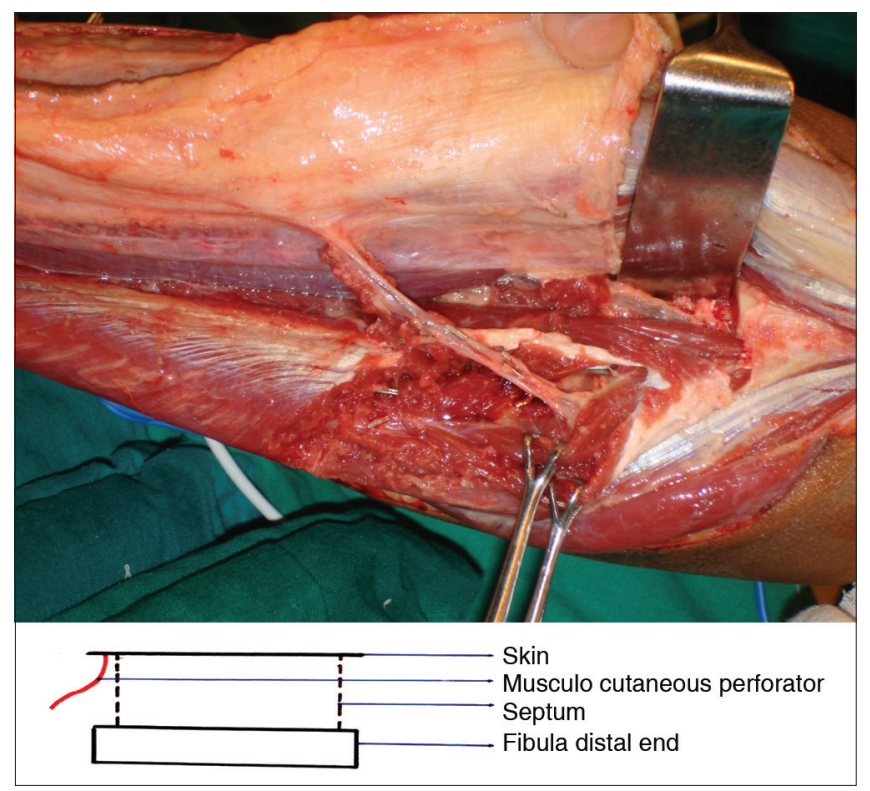

Figure 3: (a) Skin paddle receiving its sole source of blood supply from the soleus musculocutaneous perforator. There are no septocutaneous perforators seen in the septum. (b) Line diagram of Figure 3a

Indian Journal of Plastic Surgery January-April 2012 Vol 45 Issue 1 
370/386 (95.8\%) were of type A [Figures $1 \mathrm{a}$ and b]. The lower lateral leg skin had consistent blood supply via the peroneal septocutaneous perforators (1-3) and we found this region to be safe to design skin paddles.

In this group, 11 flaps were lost completely and 19 flaps were lost partially, i.e. the outer paddles used for skin cover were lost. Twenty flaps had superficial skin necrosis and were managed conservatively. The lost flaps were replaced with pectoralis major myocutaneous flap, forehead flap, nasolabial or deltopectoral flap depending on the requirement and location of the defect.

\section{Type B skin paddles}

Skin paddles receiving vascular contribution from both peroneal and posterior tibial systems ${ }^{[11]}$ were classified as type $B$ skin paddles [Figures $2 a$ and $b$ ]. These skin paddles were dependent on the contribution from the posterior tibial vessels through soleus musculocutaneous perforators for their survival. This was because the major part of the skin paddle received its blood supply from the soleus musculocutaneous perforators, which was confirmed intraoperatively by microclamping them and checking the skin paddle vascularity after releasing the tourniquet. 14/386 (3.6\%) of our skin paddles were of type B and were managed with two sets of anastomosis in the neck, one for peroneal and the other one for the posterior tibial contributions.

In this group, one flap was lost completely and was replaced with pectoralis major myocutaneous flap and forehead flap. There was one partial loss and was replaced with deltopectoral flap.

\section{Type C skin paddles}

Skin paddles receiving their vascular contribution only from the posterior tibial system ${ }^{[9,10]}$ were classified as type $C$ skin paddles [Figures $3 a$ and b]. We had $2(0.5 \%)$ such cases where the contribution came via soleus musculocutaneous perforators and were managed with two sets of anastomosis in the neck.

In this group, we did not have any loss of flaps and we did not have any contribution via septocutaneous perforators from the peroneal system.

\section{Type D skin paddles}

Skin paddles receiving vascular contribution from popliteal artery ${ }^{[13]}$ were classified as type D skin paddles. We did not encounter any such cases.

\section{RESULTS}

Skin paddle of the free fibula flap can receive contributions from axial vessels of the leg other than the peroneal system, and depending up on the contribution of the axial vessels, the skin paddles were classified into four types. We did not come across any ischaemic changes in those muscles through which the perforators were tracked till their origin, and further we observed that the survival of the external skin paddle which lies distal to the de-epithelised segment depended upon the adequacy of the subdermal plexus and not on the source or number of perforators. The drawback in our study was the inability to correlate the clinical findings with cadaveric studies.

\section{DISCUSSION}

The versatility of the free fibula flap comes from many factors and one among them is a large skin paddle with a reliable vascular supply. A large skin paddle harvested from the lateral leg provides extensive soft tissue cover in oro-mandibular reconstruction by providing both the inner oral lining and outer skin cover. In the initial studies of mandible reconstruction with free fibula flaps, the reliability of the skin paddle and its vascular supply was doubted and contradicting reports emerged. ${ }^{[1,14]}$ But over the years, with increase in use and knowledge about the anatomy, it was proved that the skin paddle of any size has a reliable vascular supply and one needs to be aware of the different contributing sources to be successful..$^{[8-13]}$ Jones et al. ${ }^{[12]}$ studied extensively about the skin paddle vascularity experimentally and clinically, and concluded that if the skin paddle is designed over the lower leg, the peroneal septocutaneous perforators alone are sufficient for the skin survival. Winters et al. ${ }^{[13]}$ conducted a similar study and found that proximal aspect of the lateral leg receives contributions from posterior tibial and even popliteal vessels through soleus musculocutaneous perforators, and therefore proximal skin paddles be added with soleus musculocutaneous perforators until the adequacy from the peroneal septocutaneous perforators is confirmed. Wong et al.$^{[8]}$ studied extensively about the soleus musculocutaneous perforators and classified them into convergent and divergent systems. In the convergent system, the soleus musculocutaneous perforators arose from the peroneal vessels, and hence were harvested with the flap, and therefore can be managed with single set of anastomosis in the neck. In the divergent system, the soleus musculocutaneous perforators arose from other 
axial leg vessels, and therefore Wong et al. concluded that they require second set of anastomosis in the neck and suggested to harvest the skin and bone components separately. In this study, other than type A skin paddles, rest all had divergent type of perforators and all of them were harvested as a single composite unit contrary to Wong et al.'s suggestions, but were managed with two sets of anastomosis in the neck. ${ }^{[10,11]}$ They concluded that septocutaneous perforators from the peroneal vessels may be absent in $5-10 \%$ of the limbs and in these cases it is preferable to look for soleus musculocutaneous perforators to salvage the skin paddle. In our study, complete absence of septocutaneous perforators was noted only in $0.5 \%(2 / 386)$ of cases, while $3.6 \%(14 / 386)$ had significant contributions from the posterior tibial vessels in addition to the contribution from the peroneal vessels.

\section{CONCLUSION}

Skin paddle of the freefibulaflap has reliablevascularsupply from the axial vessels of the leg, and though majority of them receive their vascularity from the peroneal vessels, one needs to be aware of the contribution from other axial vessels to be successful, especially in skin paddles extending proximal to the mid leg. Though we differ from Wong et al. in that flaps with divergent perforator system be harvested as two separate components, we agree with studies of Wong et al. and Winters et al. showing that soleus musculocutaneous perforators be preserved especially in skin paddles extending proximally until the contribution from the septocutaneous perforators is confirmed.

\section{ACKNOWLEDGMENTS}

Dr. Medha V. Johsi, Mr. M. P. Chandramohan and Mr. Rupesh P. Jadhav are gratefully acknowledged.

\section{REFERENCES}

1. Taylor GI, Miller GD, Hamm FJ. The free vascularised bone graft. Plast Reconstr Surg 1975;55:533.

2. Wei FC, Chen HC, Chuang CC, Noordhoff MS. Fibular osteocutaneous flap: Anatomic study and clinical application. Plast Reconstr Surg 1986;78:191-200.

3. Hidalgo DA. Fibular free flap: A new method of mandibular reconstruction. Plast Reconstr Surg 1989;84:574-85.

4. Wei FC, Seah CS, Tsai YC, Liu SJ, Tsai MS. Fibula osteoseptocutaneous flap for reconstruction of composite mandibular defects. Plast Reconstr Surg 1994;93:294-304.

5. Hidalgo DA, Rekow A. A review of 60 consecutive fibula free flap mandible reconstructions. Plast Reconstr Surg 1995;96:585-96.

6. Cordeiro PG, Disa JJ, Hidalgo DA, Hu QY. Reconstruction of mandible with osseous free flaps: A 10 year experience with 150 consecutive patients. Plast Reconstr Surg 1999;104:1314-20.

7. Yadav PS, Ahmad QG, Shankhdhar VK, Nambi GI. There is no donor side specificity of fibula free flap for complex oromandibular reconstruction. Indian J Plast Surg 2010;43:177-80.

8. Wong $\mathrm{CH}$, Tan BK, Wei FC, Song C. Use of the soleus musculocutaneous perforator for skin paddle salvage of the fibula osteoseptocutaneous flap: Anatomical study and clinical information. Plast Reconstr Surg 2007;120:1576-84.

9. Tan BK, Wong $\mathrm{CH}$. An anomalous septocutaneous perforator to the skin paddle of the fibula osteocutaneous flap originating from the posterior tibial artery. J Plast Reconstr Aesthet Surg 2009;62:690-2.

10. Yadav PS, Ahmad QG, Shankhdhar VK, Nambi GI. Successful management of free osteocutaneous fibula flap with anomalous vascularity of the skin paddle. Indian J Plast Surg 2009;42:255-7.

11. Ahmad QG, Yadav PS, Shankhdhar VK, Nambi GI. Use of fibula pedicle as a composite interposition vascular graft to salvage large skin paddle with anomalous vascular supply. Eur J Plast Surg 2011;34:413-6.

12. Jones NF, Monstrey S, Gambier BA. Reliability of the fibula osteocutaneous flap for mandibular reconstruction: Anatomical and surgical confirmation. Plast Reconstr Surg 1996;97:707-16.

13. Winters HA, de Jongh GJ. Reliability of the proximal skin paddle of the osteocutaneous free fibula flap: A prospective clinical study. Plast Recostr Surg 1999;103:846-9.

14. Schusterman MA, Reece GP, Miller MJ, Harris S. The osteo cutaneous free fibula flap: Is the skin paddle reliable? Plast Reconstr Surg 1992;90:787-93.

How to cite this article: Yadav PS, Ahmad QG, Shankhdhar VK, Nambi GI. Skin paddle vascularity of free fibula flap - A study of 386 cases and a classification based on contribution from axial vessels of the leg. Indian J Plast Surg 2012;45:58-61.

Source of Support: Nil, Conflict of Interest: None declared.

Announcement

IPhone App

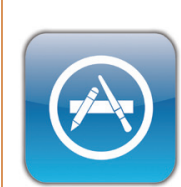

Download

iPhone, iPad application
A free application to browse and search the journal's content is now available for iPhone/iPad. The application provides "Table of Contents" of the latest issues, which are stored on the device for future offline browsing. Internet connection is required to access the back issues and search facility. The application is Compatible with iPhone, iPod touch, and iPad and Requires iOS 3.1 or later. The application can be downloaded from http://itunes.apple.com/us/app/medknow-journals/ $\mathrm{id} 458064375$ ? $\mathrm{s}=18 \mathrm{mt}=8$. For suggestions and comments do write back to us. 